\title{
The Effects of Virtuality on Agile Development
}

\author{
Karen Stendal \\ University of South-Eastern Norway and \\ NHH Norwegian School of Economics \\ karen.stendal@usn.no \\ Hanna Midttun Rostrup \\ NHH Norwegian School of Economics \\ hanna.m.rostrup@gmail.com
}

\author{
Jon Iden \\ NHH Norwegian School of Economics \\ jon.iden@nhh.no \\ Daniel Elston \\ NHH Norwegian School of Economics \\ daniel.elston@hotmail.com
}

\begin{abstract}
In this article, we explore how agile development teams are affected by transition from physical to virtual agile teamwork. To this end, we examined three agile teams at a software company, which due to Covid-19 had to change from working in a shared office space to individual home offices. We find that virtual work affects agile development in that there are fewer interactions, more written communication, more formalized relationships, and increased use of documentation. Furthermore, we find that virtual agile teams need a different style of team management. In light of this, we discuss whether a virtual context is compatible with agile development, or whether the form of work is affected so much that it no longer can be considered agile.
\end{abstract}

\section{Introduction}

In this article, we investigate whether virtual teamwork is compatible with the values and principles of agile software development. Agile software development was introduced some 20 years ago, as a response to the plan-driven, formalized, heavyweight approaches from the 1980s. Agile development is not a discrete process, but a general approach that emphasizes iterating working software code rather than formal planning, and intense collaboration over the course of the development. Close physical contact among team members is said to be necessary to ensure effective collaboration [1].

Virtual teams have been defined as groups of individuals who work together in different locations on interdependent tasks, sharing responsibility for the outcome, while relying on technology to provide most of their communication [2, 3]. The practice of using virtual teams in organizations has become popular, and it is argued that virtual work will be more common after the Covid-19 pandemic ends. Thus, it is important to organizations involved in agile development to understand how virtual work affects the work practices and performance of agile teams. The team leader has a central role in agile teams. One main task is to contribute to effective collaboration among team members, another to be a filter between the customer and the team.

Therefore, our research question is: How are agile development teams affected by transition from physical to virtual agile work?

To answer this question, we conducted a qualitative exploratory study of three agile teams at a Norwegian software development company, which because of Covid-19 transformed into virtual agile teams. Data were collected in 12 in-depth interviews with team members and team leaders, as well as observations of digital team meetings.

The study makes three contributions. First, it contributes to the literature on agile development by documenting what happens when team members work and collaborate in a virtual context. Second, the study contributes to the literature on virtual work, and the effects of virtual work on collaboration within teams. Third, the study contributes to practice by proposing measures that can remedy the disadvantages of team members not being able to interact and collaborate in a shared office location.

The remainder of the paper is structured as follows: First, we present related literature on agile development and virtual teams. Then, we introduce the research method, which is followed by the findings. Finally, we present a discussion of the findings and conclusions. 


\section{Related literature}

\subsection{Agile development and agile teams}

The term agility has been defined as "the continual readiness of an ISD method to rapidly or inherently create change, proactively or reactively embrace change, and learn from change while contributing to perceived customer value (economy, quality, and simplicity), through its collective components and relationships with its environment" [4].

As a response to the traditional waterfall approach to software development, agile development was introduced in the early 2000s. By this time, software development organizations had begun to explore more flexible approaches. Agile development suggests that software should be developed and delivered incrementally by a team, where the team members share four values [5]: close collaboration and interactions instead of formal processes and tools, a solution that works instead of documentation, close collaboration with customers instead of formal contracts, and frequent changes instead of an initial plan (Table 1).

Table 1. The four core values of the agile manifesto [5]

\begin{tabular}{|l|}
\hline Values \\
\hline Individuals and interactions over processes and tool \\
\hline Working software over comprehensive documentation \\
\hline Customer collaboration over contract negotiation \\
\hline Responding to change over following a plan \\
\hline
\end{tabular}

Although the focus should be on individuals and interactions, processes and tools cannot be overlooked [6]. Processes and tools create value when and if the team is able to take advantage of them [6]. Further, agile development does not eliminate documentation, but limits it to what is needed to perform the tasks at hand $[5,6]$. To ensure a product is developed in line with the needs of the organization or customer, the agile manifesto focuses on close collaboration with the customer throughout the development process, because the customer's needs may change during the process $[5,6]$. The essence of agile development is the ability to change and adapt to change. Although a plan is necessary for agile development, it should be lightweight and adjustable [6]. In short, typical characteristics of an agile team are close collaboration, informal and frequent communication, and continuous planning and changing.
The agile manifesto includes 12 principles, which system development projects must follow to be as agile as possible [5], as presented in Table 2.

Table 2. Twelve principles of the agile manifesto [5]

\section{Principles}

\#1 Our highest priority is to satisfy the customer through early and continuous delivery of valuable software.

\#2 Welcome changing requirements, even late in development. Agile processes harness change for the customer's competitive advantage.

\#3 Deliver working software frequently, from several weeks to several months, with a preference for the shorter timescale.

\#4 Businesspeople and developers work together daily throughout the project.

\#5 Build projects around motivated individuals. Give them the environment and support they need and trust them to get the job done.

\#6 The most efficient and effective method of conveying information to and within a development team is faceto-face conversation.

\#7 Working software is the primary measure of progress.

\#8 Agile processes promote sustainable development. The sponsors, developers, and users should be able to maintain a constant pace indefinitely.

\#9 Continuous attention to technical excellence and good design enhances agility.

\#10 Simplicity - the art of maximizing the amount of work not done - is essential.

\#11 The best architectures, requirements, and designs emerge from self-organizing teams.

\#12 At regular intervals, the team reflects on how to become more effective, and then tunes and adjusts its behavior accordingly.

For this study, we use four principles, i.e., the principles that directly address team composition, teamwork, and team leadership. These are principles $5,6,11$, and 12 .

The fifth principle focuses on the team leaders' need to find motivated team members and show them trust and autonomy in their work [5]. This means the team must be built around the right individuals with the right skills for the project. For an agile team, it is crucial that the team totally possesses the skills needed to solve the task. The sixth principle focuses on inter-team communication and the dissemination of information between members, which is best solved when team members meet face-to-face and in real-time [5]. Inter-team communication is more successful when a team is co-located [7]. The 11th principle focuses on the agile team's ability to function as a self-organizing team [5]. An agile team is required to reorganize itself multiple times during a project to solve the challenges that occur during the process [1]. The 11th principle states that agile 
project work should be organized using self-directed teams, which gives great freedom in how the task is solved. Finally, the 12th principle encourages agile teams to reflect on how to become more effective [5]. Agile teams should conduct retrospective meetings to evaluate effectiveness and reflect on how to improve their work practices [8].

Agile teams differ from traditional teams in the way they are organized and structured, where agile teams to a greater extent follow a flat structure [9]. Agile teams must be flexible and ready for change, they need to work physically closely together to minimize the cost of information sharing, and they must able to work incrementally to ensure more rapid decision making [1] and common understanding of the challenges at hand $[10,11]$. The self-organizing nature of agile teams, however, does not eliminate the need for leadership or management [1].

\subsection{Agile team management}

Agile development has been criticized concerning the lack of clarity on how an agile team should be managed [12, 13]. Although there is disagreement about the leadership roles in an agile project, researchers agree on daily management and which elements fall to the team leader of agile projects [13, 14].

A leader of an agile team needs to be inclusive and supportive [13, 14], and be a mentor and facilitator to ensure collaboration, initiative, and shared decision making within the team [14]. A team leader is required to ensure the right skills and competencies and a shared understanding among the team members for the task [13]. Leading an agile team is complex. On one hand, there is a need for overview and control, through tools and daily meetings, and on the other, the self-organizing nature of agile teams reduces the leader's controlling leadership style [13]. There is a need for balance in the team leader role $[1,13]$.

Another important role for an agile team leader is gatekeeper [13], where the team leader acts as the interface between the team members and the customer. The team leader must respond to the customer's inquiries and thus, ensure that the team can work without disruption to the greatest possible extent. Due to this role, an agile team leader must have high competences, experience, and great communication skills [13].

\subsection{Virtual teams}

Virtual teams have been defined as goal-oriented groups who meet without members being physically co-located. A virtual team collaborates independent of time and space through information and communication technology [15]. In addition, virtual teams may not be geographically separated, but the main criterion is that the collaboration is conducted mainly through information and communication technology [16]. Virtual teams use email, video conferencing, and other online-based technology as a replacement for face-to-face communication when they are not physically co-located [17].

Organizing work in virtual teams offers increased flexibility and reduced costs, and provides access to global competence [18]. However, some challenges have been identified for virtual teams. Communication is one of the main challenges [19, 20]. Communication in virtual teams is known to be asynchronous and less interactive [20]. Increased use of asynchronous communication may decrease communication quality and information transfer among team members $[19,21]$. This may lead to increased response time in information sharing as well as misunderstandings [19]. Virtual teams experience lower communication frequency and more formal communication [19], which may lead to more conflicts among virtual team members compared to teams that are co-located.

Coordination and collaboration are also identified as challenges for virtual teams [22]. Therefore, coordination and collaboration technologies, where team members can work simultaneously, plan, coordinate, and collaborate are important for virtual team success [23].

Leading a virtual team has been found to be a challenging task [24]. Lack of physical contact and less opportunity to observe team members make it difficult to control inter-team communication, facilitate coordination, and oversee work progress [25]. Establishing personal relationships and trust with team members has also been identified as a challenge [24].

\section{Research method}

To investigate the research question, we examined agile teams in a company with long experience with agile development, and where due to the Covid-19 pandemic, the agile teams had switched to a virtual form of teamwork. We chose to conduct a qualitative case study, as case studies are an appropriate approach for exploring a phenomenon within a context where there is a need for in-depth knowledge [26].

The research context for the case study is a Norwegian company, which aids organizations in 
multiple industries, in the public and private sectors, with software development. For software development, the organization uses cross-disciplinary teams, and digital tools are an important part of dayto-day operations. Since 2006, the company has applied agile development as their preferred method to software development, and all teams strive to utilize the agile approach.

\subsection{Data collection}

In this study, we followed three teams in the case organization. At the time of the data collection, all teams had moved from being physically located to working virtually. This transition happened in March 2020, because of the Covid-19 pandemic. The data collection was carried out in the fall of 2020. The teams had four to 12 team members and from the beginning had used Scrum and Kanban as their agile method.

The three teams were established in 2006, 2008, and 2018, respectively. The team members and team leaders knew each other well, except for one team leader, who had recently been hired. Figure 1 shows when the teams were formed and when they moved to working virtually.

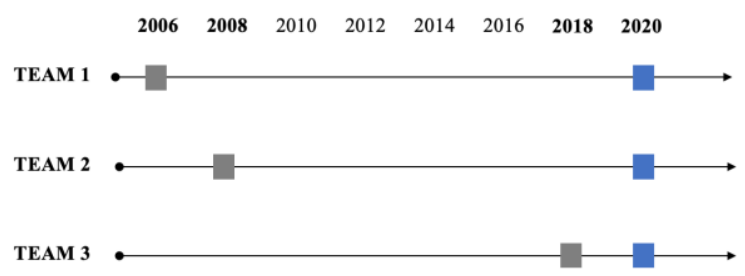

Figure 1. When the teams were formed and when they moved to working virtually

We used a strategic approach for selecting participants [27], based on three criteria: 1) The participants should be experienced with agile development, 2) we wanted to interview team leaders and team members, and 3) participation had to be voluntary.

Table 3. Overview of participants and their experience with agile development

\begin{tabular}{|l|l|l|l|}
\hline $\begin{array}{l}\text { Team } \\
\text { leaders }\end{array}$ & $\begin{array}{l}\text { Agile } \\
\text { experience }\end{array}$ & $\begin{array}{l}\text { Team } \\
\text { members }\end{array}$ & $\begin{array}{l}\text { Agile } \\
\text { experience }\end{array}$ \\
\hline Participant 3 & 2 years & Participant 1 & 6 years \\
\hline Participant 4 & 12 years & Participant 2 & 12 years \\
\hline Participant 6 & 2 years & Participant 5 & 10 years \\
\hline Participant 8 & 7 years & Participant 7 & 4 years \\
\hline & & Participant 9 & 8 years \\
\hline & & Participant 10 & 2 years \\
\hline & & Participant 11 & 3 years \\
\hline & & Participant 12 & 5 years \\
\hline
\end{tabular}

In total, interviews were conducted with 12 people. For reasons of anonymity, no further information about the case company, the teams, and the team members can be provided. Table 3 shows an overview of the participants.

To collect data, we conducted in-depth interviews and observations. Four team leaders and eight team members participated in interviews. All participants had considerable experience working with agile development. The interviews were semi-structured, based on an interview guide, one interview guide for the team leaders and one for the team members. All interviews were conducted on Microsoft Teams and lasted between 30 and 65 minutes. The interviews were recorded and transcribed. To ensure anonymity, all audio files were deleted immediately after they were transcribed, and no personal information was included in the text.

In addition to the interviews, we conducted nonparticipatory, unstructured observation of four virtual "stand up" meetings. In this way, we gained insight into how the teams worked, interacted, and collaborated virtually. During the meetings, we had our camera and microphone turned off, so that the participants were not affected by our reactions and behavior.

\subsection{Data analysis}

Through collecting and transcribing the data, we familiarized ourselves with the data. The interview data were analyzed according to the principles of thematic analysis [27], where we searched for recurring themes and patterns. We used an abductive approach [28], in which we combined and alternated between searching the data material for known topics and new topics that could emerge in the data. The analysis was performed in four steps.

In the first step, we became acquainted with the data material through two of the researchers each reading the transcripts twice, noting key points, and writing a summary of each interview. In the second step, we coded directly in the transcripts. We used in vivo coding techniques, for example, codes introduced by participants, and a priori coding techniques based on terms from existing literature [28]. In the third step, we searched for common themes, as well as relationships between the various themes. All codes were transferred to a spreadsheet, to make the analysis more efficient. To get a visual overview, a mind map was prepared with themes and key relationships between them. Finally, in step four, we reconciled the topics against the transcription, to validate the findings and to find explanations for and connections in the findings. In the next section, 
quotes are translated from Norwegian to English to best convey the original meaning.

\section{Findings}

We present findings regarding participants' experiences and their reflections concerning the transition from physical to virtual agile work. This transition is shown through four themes: communication, planning and coordination, collaboration, and management. Quotes from interviews and notes from observations are used to illustrate the findings.

\subsection{Communication}

The agile development teams used Slack and Teams even before the pandemic occurred. Slack was used for communication within the teams, while Teams was used to communicate with customers. When the teams moved to working virtually, they continued using these communication tools offered by the organization.

The transition to virtual work was experienced as easier than expected. Participants identified their previous familiarity with the communication tools as an important factor: "The big change is that everything happens through Teams. When we worked as physical teams, we used both Slack and Teams, however, much more loosely" (Participant 12).

Communication frequency was a recurring theme in the interviews. However, an effect of the transition to virtual teamwork was they communicated less within the team:

I feel that the total communication has decreased. I have difficulties to see what has become better, because the opportunity to communicate virtually we always had. One major element has been eliminated; you know, there is something about being able to just turn around and ask and discuss with somebody directly. The communication around the lunch table and over a cup of coffee is also gone in the virtual environment. (Participant 10)

One reason for the decreased communication frequency was that the barrier to contact other team members had increased. Nobody wanted to intrude. When the team was physically co-located, it was easy to determine when and if a team member was in deep concentration or if he or she had time to answer a question. When you do not see your colleagues, participants told us, you hesitate to make contact. They also told us that it was more difficult to understand each other using digital tools.
Formal meetings within a sprint, such as daily meetings, planning meetings, and evaluation meetings, were conducted as normal. It was especially the unplanned, immediate, and informal communication that was harmed, described by the participants as informal professional discussions, talking about "other things," and coffee talk. Informal chats and discussions about challenging topics, chats that normally would happen around the coffee pot, vanished. Due to this, participants reported lacking professional discussions, feeling lonely, increased distance among team members, and not feeling part of a team. This was especially true for new hires, who identified the lack of opportunities to create personal relationships with other team members as worrisome.

The findings show reduced communication frequency, especially less informal, spontaneous communication, which resulted in weaker personal relations among team members. Actions to increase informal communication had varied results.

\subsection{Planning and coordination}

The teams organized their work based on sprints with a time frame of two to four weeks. For each sprint, regular planning and coordination meetings were set up. For this, Azure DevOps and Jira were used, with digital boards adapted for agile project work. When the teams switched to working virtually, it was with the support of digital tools that had already been incorporated, and that the team members and customers were familiar with.

In the office, the teams also used white boards, which according to the participants contributed to a better overview of the task and to a common understanding within the team than the digital tools do. One team leader said that no longer using the white board for planning and coordination reduced the team's efficiency:

We discussed this yesterday. The team members felt that sometimes when they talked together it became so ineffective because they did not have the same understanding when they left the meeting... Communication became ineffective due not being able to draw it up [on the white board] and agree. (Participant 4)

Various initiatives were undertaken to find replacements for the white board. One team agreed that one participant, after a meeting, should make a sketch (figure) of what they had discussed, take a photo, and send it to the rest of the team. In another team, the team leader had started writing minutes to ensure that the team had a common understanding of what they had decided. Similar documentation needs 
also emerged in the other teams. For example, a team started setting up daily lists of what individual team members should work on. Each team member entered their work assignments in Slack, and after the team leader coordinated the content, the list was distributed to all team members.

The findings show there was little change in the use of digital tools for planning and coordination. However, the absence of physical visualization aids makes it difficult for team members to have an overview of what is happening in the project, and not least what the team members are doing. To compensate, the need for documentation increased.

\subsection{Collaboration}

The participants said that the transition from colocation to a virtual form of work led to less collaboration. Moving from physical to virtual teams led to increased individual work. The participants assessed the change differently: Most were negative, but some thought the change also had positive aspects. Some team members experienced being able to concentrate better working alone in their home offices. Due to decreased unplanned communication, as presented, some participants experienced fewer interruptions by fellow team members. Generally, however, participants reported that the reduced contact and collaboration were challenging:

What I see is that being able to easily talk to people becomes a challenge. A lot of the collaboration happens during the day where you turn in your chair and ask the person sitting next to you. You will not get that anymore.... Collaboration takes longer. It is a bit strenuous sometimes. (Participant 12)

Team leaders indicated that personal relations, transparency, and trust were needed as building blocks for good collaboration. To improve collaboration, each team had intensified their use of digital collaboration tools, and some had adopted new ones. The chat features in Slack and Teams were increasingly used to collaborate on what were referred to as "small issues." For more comprehensive tasks, team members collaborated using Teams meetings. One team used Live Share for pair programming. A participant commented:

If I have an idea about how I want to solve something, and he is in control, I do not have to describe it word for word. I can write it up and ask what he thinks as we are working on it. Collaboration becomes easier. When the tools work properly, it makes a big difference to the virtual collaboration. (Participant 10)
The findings show collaboration in virtual teams led to more individual work, which, for some, could lead to better concentration. Nevertheless, participants agreed that it was more difficult to collaborate when working together virtually. Some referred to the collaboration as now "more cumbersome" because it requires more to contact a colleague when you are not co-located. Others commented that when using digital tools, one must be more aware of how one presents oneself, in writing and orally, which makes collaborating more demanding. The team leaders said that digital meetings make it more challenging to understand how team members react to what is said, and whether the team has really reached agreement or a common understanding of the tasks. The main conclusion is that, despite increased use of digital collaboration tools, working virtually reduced collaboration in the three agile teams. The team members were less likely to contact each other, and the collaboration became more formal.

\subsection{Management}

A team leader described the team leaders' role for agile teams in the company as follows:

It is to ensure that the team members have the basic conditions needed to do a proper job. And I am a buffer between the customer and them, so that they can work as much as possible with what they are going to do without being unnecessary disturbed. I also facilitate their work, so they are productive. (Participant 3).

From the interviews, it emerged that the team leaders experienced their role as more critical for the team when working virtually than when they were colocated. The team leaders pointed out four issues. First, the leader's role as chair of the meeting became more important. Team leaders felt they needed to be more active, ask team members what they are working on, raise issues that no one really wanted to talk about, make sure the meetings are well organized and conducted, and take on the role of chair to ensure that everyone has a say.

Second, the team leader must, to a greater extent, act as a liaison between the team members outside the regular meetings. When you are not co-located, there is a need for someone who facilitates contact among team members. A team leader described this as follows:

For example, the team member who encounters a problem and need help to arrange a meeting with another team member who can help. Because you see that it will not happen by itself. He will just sit scratching his head. Maybe a week without 
getting in touch because he does not see how to do it. [...] You must go and talk to people, and make sure that people are talking to each other. (Participant 4)

Third, the team leader became more involved in the contact between the teams and the customers. In agile project work, the team leader must act as a gatekeeper between the customer and the team. When the teams are co-located, the team leader is located so that he or she can observe at any time when a customer arrives. When the teams switched to virtual work, this became more difficult, and sometimes, impossible to manage. The communication channels between the customer and the team increased. Many team members explained that customers bypassed the team leader and contacted them directly. This was considered problematic. First, it made coordination within the team more difficult, and second, it could lead to duplication of work as different people, without the team leader's management, could work on the same tasks. A team member described this as follows:

Earlier, the team leader sat in such a position in the room that no one could get in or out without passing him. So, you lose it a bit now, customers can send emails or Teams messages directly. I think the team leader must take care to ensure that there is not too much direct contact between the customer and the developers. In the virtual, you may not have the overview that you usually have. (Participant 7).

In an agile virtual team, the need for the team leader to be aware of the customer's needs and priorities and bring them to the team increased.

Fourth, to remedy the lack of social contact, the team leader must initiate social activities that facilitate informal gatherings in the teams. A team member stated:

The team leader is more important. More essential. It's a bit like sticking your head away. If you are sitting in an open landscape, as we did before, then just talk to each other. [...]. The work the team leader is doing now in the virtual setting is much more important, I think. (Participant 11)

The team leaders stated that their role as a facilitator in virtual teamwork is demanding. One pointed out that virtual work had led to more meetings than when they were co-located:

I have a lot of meetings because everything must be meetings [...] Because you cannot go past people and ask how it goes [...] I facilitate most meetings myself; there are very few meetings I am passive in. (Participant 4 ).

Team leaders also found it challenging to identify team members' individual needs for support.
Inconsistency in camera and microphone use during meetings made it difficult to observe non-verbal signals. Team leaders also identified team members' personal relations as an indication of how involved the team leader had to be. In addition, the team leader who did not know their team well before going virtual found it especially challenging to establish these personal relations.

The main conclusion is that virtual agile development challenged the traditional agile team leader's role and led to a more active, and sometimes, a more governing role. Team leaders had to take a more active role to ensure and facilitate informal communication among team members, especially for new members. The team leader also had to make himself or herself more visible and active as a gatekeeper between the team and the customer. The findings show an increased need for management in virtual agile teams.

\section{Discussion}

The move from physically co-located agile teams to virtual agile teams was shown to be challenging. In this section, we discuss the research questions.

\subsection{The effects of virtuality on agile development}

Our research question is: How are agile development teams affected by transition from physical to virtual agile work?

Through a review of the literature, we found four central principles important for efficiency in agile teams. Our analysis showed that all of them are affected when agile teams work virtually. We identified four shifts in agile development practice, shown in Table 4.

Table 4. The effects of virtuality on agile development

\begin{tabular}{|c|c|}
\hline $\begin{array}{c}\text { Principles for effective } \\
\text { agile teams }\end{array}$ & $\begin{array}{c}\text { Virtual effects on agile } \\
\text { development }\end{array}$ \\
\hline $\begin{array}{c}\text { Face-to-face } \\
\text { communications }\end{array}$ & $\begin{array}{c}\text { Written communication } \\
\text { increased }\end{array}$ \\
\hline Informal relations & Relations were more formal \\
\hline Frequent interactions & Interactions decreased \\
\hline $\begin{array}{c}\text { Working software over } \\
\text { comprehensive } \\
\text { documentation }\end{array}$ & Documentation increased \\
\hline
\end{tabular}

5.1.1. From face-to-face to increased written communication. According to agile development, oral face-to-face communication is the most efficient form to ensure information sharing among team members [5]. Face-to-face communications 
contribute to frequent, fast, and flexible informationsharing capabilities [22].

Our analysis showed, unsurprisingly, that working virtually led to a decrease in communication among team members and an increase in written communication. Unplanned, spontaneous communication suffered especially. Compensation for this development was sought using digital tools (chat and video calls), but these channels did not seem to fully cover the absence of eye contact. According to the literature, this is an expected consequence of virtual work [20]. Researchers have shown that communication in virtual teams is characterized as more written and asynchronous compared to teams that are co-located. Although this development was expected, it violates the first of the four agile principles.

5.1.2. From informal to formal relations. The second principle is that the relations among team members should be informal. The goal is to ensure transparency and closeness, which is important for a team that collaborates to meet short deadlines [1]. Informal relations intend to contribute to good information sharing and ensure continuous understanding within the team about tasks to be completed during each sprint [7, 29].

Our analysis showed the relations among team members became more formal after they transitioned to a virtual work environment. We found that members' spontaneous oral communication was replaced with more thoughtful written (and to some extent, oral) communication, and that the use of prescheduled meetings increased. The teams wanted to mitigate the effect of more formalized relations, but not being co-located made this difficult. This development goes against the second of the four central agile principles.

5.1.3. From frequent to fewer interactions. Agile development emphasizes frequent and close contact among team members $[4,5]$. In line with face-to-face and informal communication, frequent interactions are identified as a key element of agile teams. The goal is to ensure information sharing and a common understanding among team members $[22,30]$.

Our analysis indicated the number of interactions among members of the agile teams decreased when they transitioned to virtual work. The team had fewer contact points and communicated less. This development is correlated with other issues, such as more formalized relations and more written communication, which is related to the higher threshold for contacting each other.
Our findings compare to previous research that explored what happens to teams that are not agile when they start working virtually $[2,20]$. Although this change was expected, it breaks with the third agile principle.

\subsubsection{From working software over documentation} to increased documentation. The fourth key principle of the agile manifesto is "working software over comprehensive documentation" [5]. This is an important element that emphasizes the requirement of frequent interactions with informal, face-to-face contact, instead of spending time documenting. Our analysis showed that the use of documentation increased after the team members started working from home offices. The teams found it difficult to communicate, plan, and coordinate each other's work without written documentation. The use of digital tools did not compensate for the loss of co-location and the reduction in interactions. Going against the agile development's downgrading of documentation, we found an increase in documentation when the agile teams became virtual agile teams.

Our initial literature review showed that agile teams are most effective when team members are physically co-located. Being co-located ensures that the team members can see each other when they communicate, that they establish close and trustworthy relationships, and that they can contact each other immediately when something occurs, which, in turn, means that the team members can focus on the product rather than on extensive documentation.

Our study showed that all these key principles are affected when agile teams work virtually. We found that the transition to a virtual way of working leads to more written communication, more formalized relations, fewer interactions, and increased use of documentation. Our analysis showed there is a need to question whether virtual agile teams can be considered agile at all. Or put another way, does the expected transition to more home office work for all after the Covid-19 pandemic mean that the agile manifesto must be rewritten? Will the increased dispersed locations of team members lead to a new version of agile development?

5.2. The team leader's role in virtual agile teams. Our analysis showed that the need for management increases for virtual agile teams, which is in line with research on virtual work that showed virtual work creates unavoidable management challenges [31]. However, the development may contradict agile teams being self-organized, self-directed, and autonomous $[1,10]$. The change may contribute to a 
shift from the ideal of a supportive team leader to a more controlling team leader [13]. We assume that the challenges posed by virtual workplace greater demands on the team leader's character traits, including the ability to steer and direct.

It has been argued that the team leader in an agile team should support and provide guidance to ensure efficiency [14]. Our analysis showed that these tasks are even more important for virtual agile teams. The transition to virtual agile teams made the gatekeeper role [13] challenging. With the introduction of multiple communication channels, the team leader found it hard to protect the team from customer communication.

Our analysis identified that the team leader must focus more on communication and collaboration among team members compared to when the team was co-located. The findings are supported by virtual management research, which showed that virtual team leaders must spend a considerable amount of time to ensure efficiency in their teams [31].

\section{Contribution and implications}

Literature on virtual agile teams is limited. The literature that does exist focuses on how traditional virtual teams incorporate agile practices, and not how agile development teams are affected by transition from physical to virtual agile work [32-34]. Thus, this research contributes to the research on agile teams in a virtual context.

Although these findings correlate with previous research concerning the requirement of common understanding of tasks, solutions, and issues in virtual teams [33], we showed that this is difficult for an agile team working in a virtual context.

Although previous research on virtual teamwork considered challenges such as those we found in this study, few researchers have evaluated whether virtual work is compatible with the principles of agile development. The present research shows that key elements of agile development are affected by being conducted virtually. We found these effects to be to a degree that should raise questions about whether virtual agile teams are less efficient than physical agile teams. We also question whether virtual agile teams can be considered agile when considering the effects virtuality has on the key agile elements.

This study also contributes by showing the increased need for management of virtual agile teams. Moving to a virtual team setting requires the team leaders, as well as the team members, to adjust their behavior. We also contribute to the discussion on how a team leader should work strategically to reduce the negative effects of virtuality on agile development.

This study was conducted in one organization and six months after they went virtual, which represent a limitation. Future research in this area should include a longitudinal study of the effects of virtuality on agile teams. Such a study can explore whether a virtual team can be agile, and whether the short-term effects shown in this study fade over time. Moreover, the team leader's role in a virtual agile team over time should also be investigated further.

\section{Conclusion}

Agile development has become a well-established approach for software development [29], and digital platforms have increased the formation of virtual teams $[18,35]$. The agile teams included in this research transitioned from a physical to a virtual work environment in March 2020. In this study, we found that the transition to virtual agile teams affected agile development in four dimensions: Written communication increased, the relations between team members were more formal, the number of interactions among team members decreased, and the use of written documentation increased. In addition, going virtual led to decreased and slower information sharing, and increased challenges in establishing a common understanding within the teams.

We also concluded there is an intensified need for a more involved team leader when agile teams transform into virtual agile teams. The team leader must step into a more active management role and continuously facilitate effective information sharing and collaboration not only among team members but also with the customer. Tools that have been shown to compensate to a certain extent for the lack of physical co-location include videocalls, virtual break rooms and meeting points, and visual tools.

Based on these findings and discussion, we are critical of moving agile teams virtual. We question whether virtual agile teams can be considered agile as described in the agile manifesto.

\section{References}

[1] Cockburn, A. and J. Highsmith, Agile software development, the people factor. Computer, 2001. 34(11): p. 131-133.

[2] De Guinea, A.O., J. Webster, and D.S. Staples, A metaanalysis of the consequences of virtualness on team functioning. Information \& management, 2012. 49(6): p. 301-308. 
[3] Dreesen, T., et al. Beyond the Border: A comparative literature review on communication practices for agile global outsourced software development projects. in 2016 49th Hawaii International Conference on System Sciences (HICSS). 2016. IEEE.

[4] Conboy, K., Agility from first principles: Reconstructing the concept of agility in information systems development. Information systems research, 2009. 20(3): p. 329-354.

[5] Fowler, M. and J. Highsmith, The agile manifesto. Software Development, 2001. 9(8): p. 28-35.

[6] Dingsøyr, T., et al., A decade of agile methodologies: Towards explaining agile software development. 2012, Elsevier.

[7] Highsmith, J. and A. Cockburn, Agile software development: The business of innovation. Computer, 2001. 34(9): p. 120-127.

[8] Highsmith, J., What is Agile Software Development?\|, October 2002. Crosstalk: Journal of Defense Software Engineering, 2002: p. 4-9.

[9] Whitworth, E. and R. Biddle. The Social Nature of Agile Teams. in Agile 2007 (AGILE 2007). 2007.

[10] Dybå, T. and T. Dingsøyr, Empirical studies of agile software development: A systematic review. Information and software technology, 2008. 50(9-10): p. 833-859.

[11] Lindsjørn, Y., et al., Teamwork quality and project success in software development: A survey of agile development teams. Journal of Systems and Software, 2016. 122: p. 274-286.

[12] Maruping, L.M., V. Venkatesh, and R. Agarwal, A control theory perspective on agile methodology use and changing user requirements. Information Systems Research, 2009. 20(3): p. 377-399.

[13] Taylor, K.J., Adopting Agile software development: the project manager experience. Information Technology \& People, 2016.

[14] Coram, M. and S. Bohner. The impact of agile methods on software project management. in 12th IEEE International Conference and Workshops on the Engineering of ComputerBased Systems (ECBS'05). 2005. IEEE.

[15] Thompson, L.L., Making the Team: A guide for Managers. 5th ed. 2015: Pearson Education.

[16] Bell, B.S. and S.W. Kozlowski, A typology of virtual teams: Implications for effective leadership. Group \& organization management, 2002. 27(1): p. 14-49.

[17] Larson, B.Z., S.R. Vroman, and E.E. Makarius, A guide to managing your (newly) remote workers. Harvard Business Review, 2020. 18.

[18] Dulebohn, J.H. and J.E. Hoch, Virtual teams in organizations. Human Resource Management Review, 2017. 27(4): p. 569-574.

[19] Marlow, S.L., C.N. Lacerenza, and E. Salas, Communication in virtual teams: A conceptual framework and research agenda. Human Resource Management Review, 2017. 27(4): p. 575-589.
[20] Purvanova, R.K., Face-to-face versus virtual teams: What have we really learned? The PsychologistManager Journal, 2014. 17(1): p. 2.

[21] Hossain, E., et al. Risk identification and mitigation processes for using scrum in global software development: A conceptual framework. in 2009 16th Asia-Pacific Software Engineering Conference. 2009. IEEE.

[22] Sharp, H. and H. Robinson, Three 'C's of agile practice: collaboration, co-ordination and communication, in Agile software development. 2010, Springer. p. 61-85.

[23] Walsh, T., Virtual team success with the power of technology advancements, in Advances in the Technology of Managing People: Contemporary Issues in Business. 2019, Emerald Publishing Limited.

[24] Hertel, G., S. Geister, and U. Konradt, Managing virtual teams: A review of current empirical research. Human resource management review, 2005. 15(1): p. 69-95.

[25] Morrison-Smith, S. and J. Ruiz, Challenges and barriers in virtual teams: a literature review. SN Applied Sciences, 2020. 2: p. 1-33.

[26] Dubois, A. and L.-E. Gadde, Systematic combining: an abductive approach to case research. Journal of business research, 2002. 55(7): p. 553-560.

[27] Patton, M.Q., Qualitative Research \& Evaluation Methods. 3rd ed. 2002: SAGE Publications.

[28] Saunders, M., P. Lewis, and A. Thornhill, Research methods for business students. 2009: Pearson education.

[29] Zavyalova, E., D. Sokolov, and A. Lisovskaya, Agile vs traditional project management approaches. International Journal of Organizational Analysis, 2020.

[30] Yu, X. and S. Petter, Understanding agile software development practices using shared mental models theory. Information and software technology, 2014. 56(8): p. 911-921.

[31] Hoch, J.E. and S.W. Kozlowski, Leading virtual teams: Hierarchical leadership, structural supports, and shared team leadership. Journal of applied psychology, 2014. 99(3): p. 390.

[32] Moe, N.B., et al. Coaching a global agile virtual team. in 2015 IEEE 10th International Conference on Global Software Engineering. 2015. IEEE.

[33] Moe, N.B., et al. Enabling knowledge sharing in agile virtual teams. in 2016 IEEE 11th International Conference on Global Software Engineering (ICGSE). 2016. IEEE.

[34] Shrivastava, S.V. and U. Rathod, A risk management framework for distributed agile projects. Information and software technology, 2017. 85: p. 1-15.

[35] Schwarzmüller, T., et al., How does the digital transformation affect organizations? Key themes of change in work design and leadership. mrev management revue, 2018. 29(2): p. 114-138. 